\section{Spatial and temporal factors in masking by edges and disks****}

\author{
M. L. MATTHEWS \\ University of Guelph, Guelph, Ontario, Canada
}

Increment threshold of a small test probe is found to be elevated by backgrounds of a disk, bar, or luminance step. The spatial parameters that produce maximum masking are found to be essentially similar with the three types of background. The time course of masking is also found to be similar for the disk and bar background. It is suggested that a fundamentally similar type of visual processing underlies each of the masking situations.

Several studies have shown that the luminance threshold of a small test probe may be influenced by the immediate presence of a border (Fiorentini, Jeanne, \& Toraldo di Francia, 1955; Matthews, 1966), a double border, that is, a bar that has either higher or lower luminance than its background (Teller, 1968), or a background disk (Westheimer, 1965, 1967). The aim of the experiments described below is to examine the assumption that such results may be seen as different manifestations of one particular type of spatio-temporal processing activity that underlies each of the stimulus situations.

A scrutiny of some of the basic findings from each of the above experimental situations reveals some striking similarities in the mode of action of the background (single border, bar or disk) on the threshold of the test probe. In the case of the single border, that is, a sharp luminance step between two uniform fields of markedly different luminance, the closer the test probe is presented to the border in the field of higher luminance the greater the elevation of threshold. Reducing the exposure time appears to reduce progressively the peak threshold adjacent to the border (Novak \& Sperling, 1963 Matthews, 1966). Reducing the luminance and/or contrast also progressively attenuates the peak threshold (Fiorentini \& Zoli, 1966). In the case of the double border, Teller (1968) has shown that the threshold of a small test probe centered on a black bar is elevated progressively as the width of the bar is reduced. A reduction in the luminance of the bar leads to a decrease in the relative amount of threshold elevation. In the case of disks, Westheimer (1965, 1967) has systematically investigated a paradign of

\footnotetext{
* This paper was presented in part to the Optical Society of America at the Annual Meeting, Chicago. October 1969.

**This rescarch was supported by National Revearch Council of Canada Grant No. APA0316.
}

Ratoosh and Graham (1951), in which the threshold of a test probe centered on a disk is found to vary as a function of the diameter of the disk. At small disk sizes, threshold increases until a point is reached when further increases in diameter of the disk reduces threshold; further increases in diameter have no observable effect upon the probe threshold, i.e., threshold remains constant. Again, the threshold elevation effect is reduced at lower disk luminances and with short exposure durations.

The main feature of these experiments is that in each case the test-probe threshold is increasingly elevated with closer proximity to a border or luminance gradient. no matter whether there is one border or two. or if the border is curved. Other similarities that should be noted are the reduction in threshold elevation with lower luminances and shorter stimulus exposures and the with peripheral presentation. In addition, threshold is found to be affected are of the same order (approximately between 3 and $20 \mathrm{~min}$ separation between border and probs in the fovea) for each of the conditions.

The main problem with this analysis is that there is no exact repetition of experimental details between the three types of approach to suggest that there is complete justification for assuming that the results can be treated as a reflection of one type of spatiotemporal interaction. Thus, the following experiments were designed to follow systematically the responses of two Ss to each of the three paradigms, while luminance is held constant and relevant spatial and temporal factors are manipulated across conditions.

\section{EXPERIMENT 1}

Westheimer's (1967) basic experiment was repeated initially with my Ss, since there was some evidence (Teller, 1969) that individual differences would produce some idiosyncracies in the spatial response function

The Ss viewed a dim uniform field with increased masking effect that is obtained the spatial configurations within which central fixation; the center of the field was illuminated for I sec with a disk in the center of which a small test probe was located. The task was to set the test probe to threshold. The independent variable was the diameter of the disk.

The apparatus used was a modified Scientific Prototype three-channel tachistoscope. While such instruments give excellent timing properties (when adequately monitored), they are known to vary in their luminance output particularly as a function of warm-up time, ambient temperature, and nature of the stimulus program (Siegel, 1968). Care was taken to ensure that luminance had stabilized before the onset of each experimental session as indicated by a Spectra Brightness Spot Meter; in addition, at frequent intervals during the session, the luminance was checked to determine if drift had occurred. Luminance control was obtained by the insertion of neutral filters into the necessary channels.

$A$ head and chin rest were mounted at the viewing aperture of the tachistoscope to position the S's head. Ss performed the task monocularly with the preferred eye. An adapting field, size $6 \times 9 \mathrm{deg}$ and luminance of $1.9 \log \mathrm{mL}$, was first seen by the $S$; slightly above the center of this field, a dim, red fixation marker in the form of a small pointer angled downwards was located. Ss were instructed to fixate at the tip of this pointer, that is, at the center of the adapting field. Ss were required to press a button that extinguished the adapting field and simultaneously illuminated the test disk, which was presented in the center of a background field of $\overline{3} .5 \log \mathrm{mL}$. The luminance of the disk was $1.56 \log \mathrm{mL}$, and its diameter could be varied in increments of $1 \mathrm{~min}$ of arc, from 3 to $25 \mathrm{~min}$. The disk was obtainyd by transillumination of an accurately drilled hole in a sheet of mild steel placed in a second channel of the tachistoscope. The disk was presented for I sec. A small 2.5.min test probe was located at the center of the disk and was presented for the total disk duration. The test probe was delivered through the third channel of the system. At the offset of the disk and probe, the initial adapting field was again visible.

The following psychophysical procedure was adopted. With the test probe at a given luminance set by $E$, the $S$ would initiate the trial by pushing the button that led to the presentation of the disk and probe; the $S$ then reported at the offset of the disk whether the probe had been seen or not. The luminance of the probe was varied randomly by $E$ between trials, using a basic minimum step size of $0.06 \mathrm{log}$ units; blank or catch trials, that is, trials on which the 


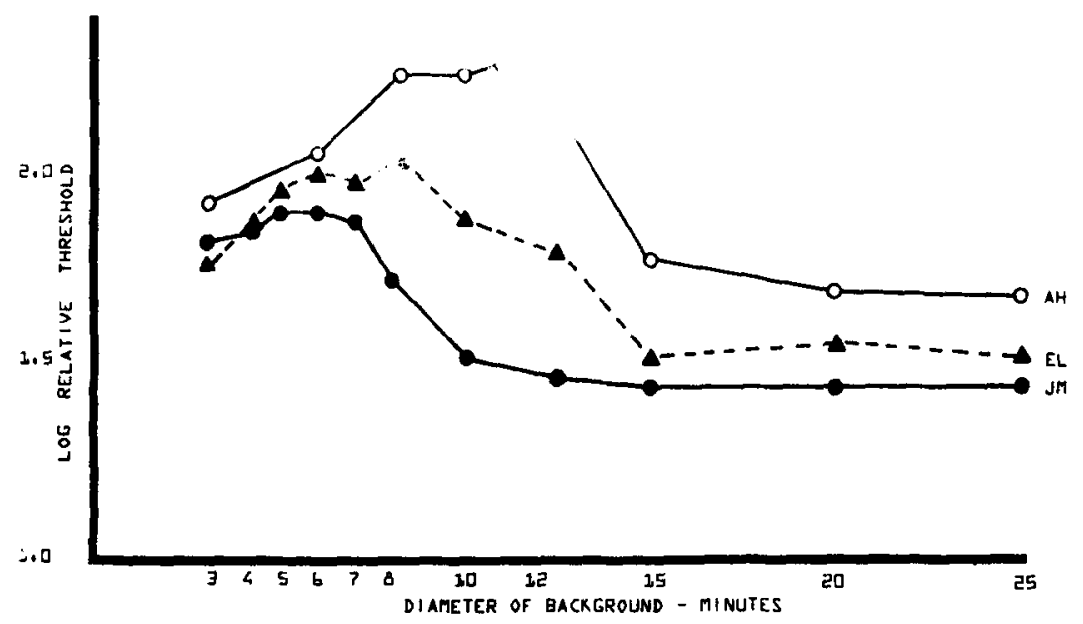

channel presenting the probe was not illuminated, were also presented to determine if criterion shifts were present across conditions. Thirty trials were used to determine threshold for each disk diameter; five of the presentations were catch trials. Threshold was considered as the luminance that gave $50 \%$ visibility of the test probe. In general, Ss would perform 15 determinations for each disk size in a single session, completing the data with the final 15 trials on a subsequent date. Sessions lasted approximately $30.45 \mathrm{~min}$. The diameter of the disks was presented in a random order to minimize progressive errors. Three Ss were used initially, two of whom had no previous experience in psychophysical judgments.

\section{Results}

Figure 1 shows the results of this experiment. For each $S$, threshold luminance (as defined above) is plotied on the ordinate as a function of the diameter of the disk. Standard deviations for the three $S$ s ranged from 0.04 to $0.09 \mathrm{log}$ units. False-alarm rates were generally found to be zero; where false alarms had

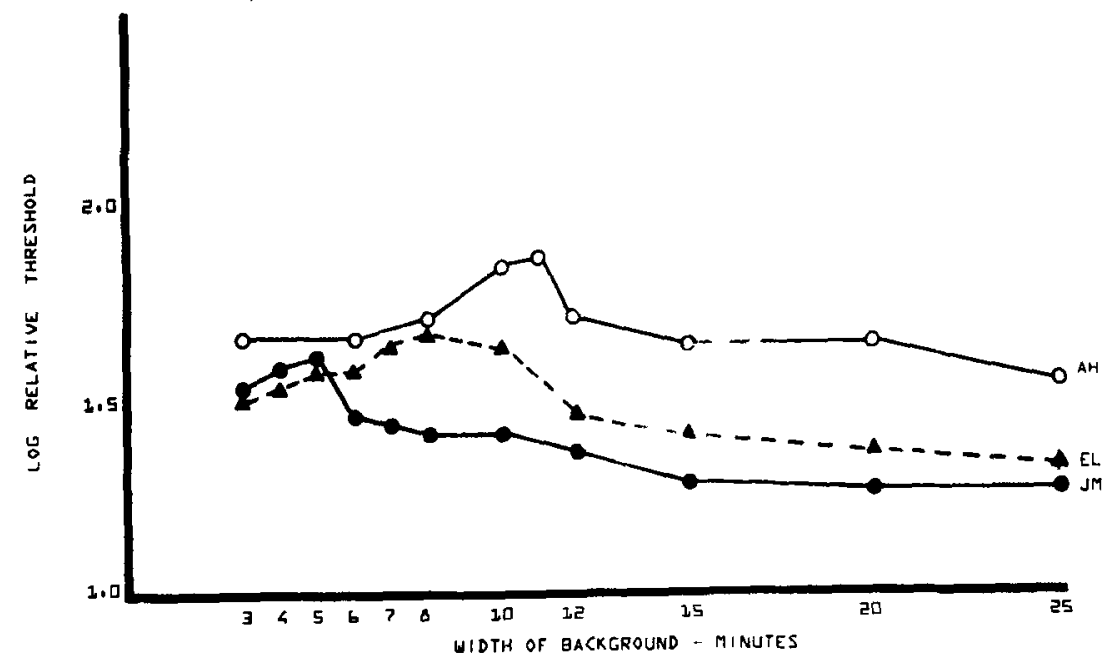

Fig. 1. Increment threshold of test probe as a function of background diameter of disk for three Ss.

does take place in the center of a dark bar, the main questions to be asked here are: (1) Is the magnitude of the elevation found with disks of the same order for bars? (2) Are the maximum elevations of probe threshold to be found with bars of a similar angular subtense as the disks that gave maximum elevation in the first experiment?

The apparatus used in this experiment was the same as in Experiment 1, except that Channel 2 now contained an adjustable mask that enabled presentation of a vertical bright bar of variable width in place of the disk used previously. The luminance of the bar was $1.56 \log \mathrm{mL}$. The mask was constructed from two sliding pieces of steel whose adjacent edges were formed by the attachment of suitable size sections cut from a precision steel rule. This ensured that when the edges were very close together the central stripe that was transilluminated between them appeared of uniform width throughout its entire length. All other experimental conditions were the same as Experiment 1; in this case, the test probe was located in the center of a vertical bar, and thresholds for the probe were again established for the three Ss as a function of bar width.

\section{Results}

Figure 2 shows the results for this experiment. The data have been plotted in a similar manner to Fig. 1, with the exception that the abscissa now represents the angular width of the bar.

The results show clearly that the threshold elevation effect is markedly reduced with bar stimuli; in this case, the elevation is about $0.3-0.35 \log$ units above minimum bar threshold, whereas for the disks the order of elevation was about 0.45 to $0.6 \log$ units. However, the angular dimensions of the two types of background that give maximum threshold elevation are remarkably similar within $S$ s. In the case of bars, the optimum width for JM was $5 \mathrm{~min}$, for EL approximately $8 \mathrm{~min}$, and for $\mathrm{AH}$ about $11 \mathrm{~min}$. These similarities will be discussed more fully in the conclusion, but it should be noted at this point that thesc data alone would tend to indicate that thresholds measured at the center of both bars and disks appear to be dependent upon the same mechanism.

Fig. 2. Increment threshold of test probe as a function of the width of background bar for three Ss. 


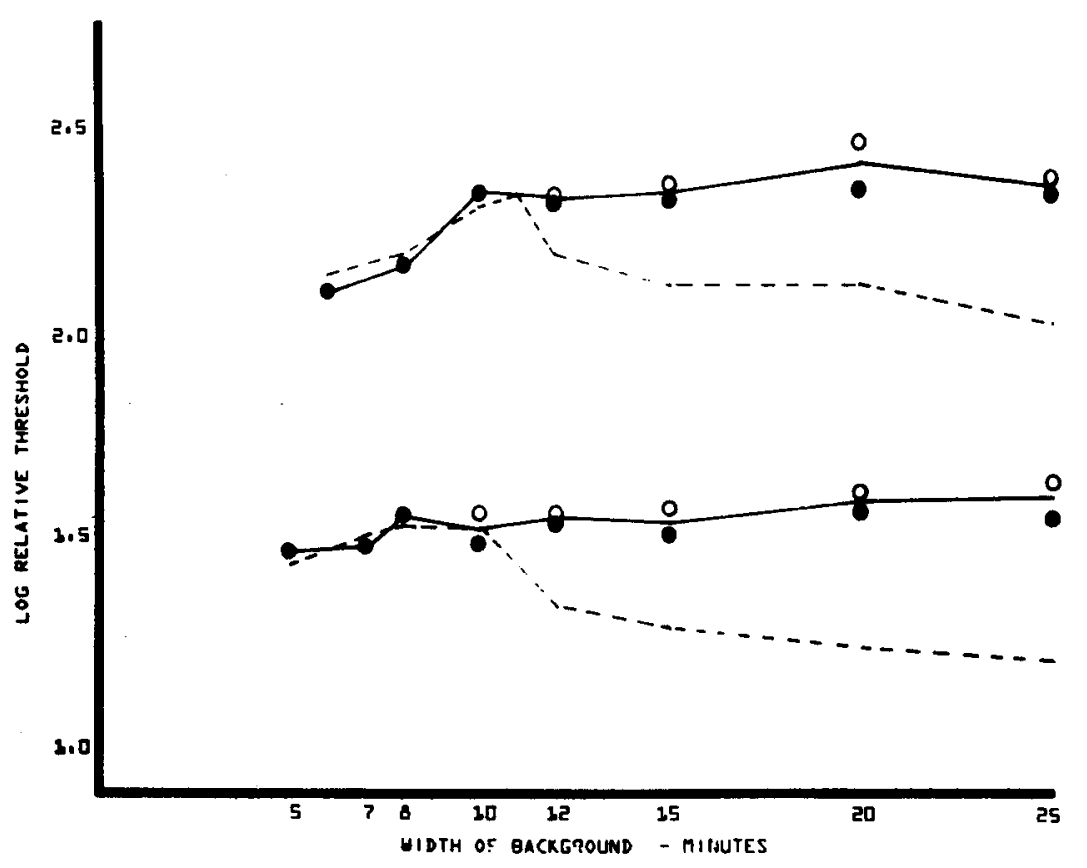

EXPERIMENT 3

The next step in the development of this analysis should be the comparison of thresholds obtained in Experiment 2 with threshold obtained adjacent to a sharp luminance gradient, that is, a double-vs single-border comparison. Evidence cited previously indicates that probe threshold is elevated in the immediate vicinity of a sharp step in luminance; thus, when two edges are widely separated spatially, as with wide bars, two threshold peaks will be obtained, one adjacent to each edge. However, it is not clear what happens to these peaks when the bar is made progressively narrower, bringing the edges closer together. Experiment 2 has shown that the threshold at the center of the bar is elevated, with small separations between the two edges. Here the question is, what is the relationship between threshold at the center of the bar and that at the edge when bar width is reduced? Clearly, if simple summation occurs, then one would expect that center peak threshold at narrow bar widths to be simply a summation of the two peaks that are obtained at the edge with wider bars. On the other hand. if there is no summation between the two edge peaks as they gradually draw spatially closer together, then the threshold obtaıned at the center with small bar widths should be of an equivalent order to that obtained adjacent to either of the single edges at wider bar widths. Of course, other outcomes are obviously possible. such as nonlinear summation, and should not be excluded from consideration.

Evidence is therefore needed to show how threshold elevation adjacent to a single border is related to the double
Fig. 3. Increment threshold of test probe located adjacent to left-hand edge (solid circles) and right-hand edge (open circles) of bar as a function of the width of the bar. Overlapping data points given by single symbol only. For comparison, broken line indicates threshold obtained with the probe centered on the bar, and is replotted from Fig. 2. Data are given for two Ss; functions of AH have been elevated by $0.6 \log$ units for clarity.

the bar. For each $S$, the response at the edges is found not be be symmetrical; in both cases, the threshold obtained at the left-hand edge is consistently lower, although the effect is within the range of the standard deviation obtained. The graphs show no indication of summation of threshold between the two edges at narrow bar widths and show that the threshold elevation with the test probe in the center of the bar is of the same order as the threshold of the probe adjacent to the edge at wider bar widths. Thus, the threshold elevating effect found at the center of narrow bars may be regarded as equivalent to the threshold elevation found at a single edge for the luminance levels used in this. experiment. The failure to find any summation between the edge responses may be somewhat surprising; however, I believe that this may be due to the fact that relatively low luminance levels were used in these experiments. There is some evidence to suggest that summation may be found when either the contrast or the luminance of the bar are higher (Matthews, 1967).

The asymmetry of the edge response is not easy to explain. Since the direction of the response is the same for the two Ss, it could be interpreted as either an uncontrolled artifact in the experimental situation or due to some fundamental asymmetry of processing within the visual system. While microdensitometric readings were not taken across each of the edges to check the possibility of the effect being due to small differences in luminance distribution, it should be noted that the effect is obtained at a wide variety of edge separations, hence any variation in luminance that would have been required to produce this effect over such wide spatial areas would have been recorded by the calibration measurements taken with the photometer described previously. At the present time, I would favor the possibility that the results reflect asymmetry of processing and, although this particular effect is very small, there is some evidence that asymmetries in processing can occur in similar situations (Cutrona \& Richards, 1969). 
Fig. 4. Increment threshold of test probe as a function of time between presentation of probe and background onset. Background is illuminated at time 0 , extinguished at $1.0 \mathrm{sec}$. Functions are plotted for three types of background: extended uniform field (solid circles), bar (open circles), and disk (squares). Dimensions of backgrounds are given in text. A plot of one standard deviation is shown on the right for each background condition. Data for $\mathbf{S}$ AH.

\section{DISCUSSION OF EXPERIMENTS 1, 2, AND 3}

The previous experiments have all investigated situations in which the threshold of a small test probe may be independent of the luminance on which it is measured and raised by the presence of an adjacent contour or contours in the visual field. The experiments show that there is a fundamental similarity in the spatial-response characteristics indicated by the probe threshold in situations in which the background responsible for threshold elevation is a disk, bar, or single luminance step. The essential characteristics of the spatial response function are found to be the same for a given $S$ between experimental conditions; the shapes of the functions and background widths responsible for maximum threshold clevation are found to differ considerably between Ss. A discussion of the type of information processing that may be responsible for these effects will be postponed until the final discussion.

Since the spatial parameters underlying the three experimental paradigms described are found to be substantially the same, a question might be asked as to whether the temporal characteristics are also the same. If my argument is correct, that one particular type of processing is involved. this should also be reflected in the time course of the spatial interactions.

While the literature is saturated with experiments on temporal factors in masking in general, few are applicable to this situation. The particular technique employed in this study to examine temporal parameters follows a design of Crawford (1947). Crawford found that the increment threshold of a disk upon a background of a larger disk was dependent on the temporal relationship between the iwo disks. Threshold was found to be elevated prior to the onset of the background, elevated maximally at the moment of onset, to gradually decrease as the background remained illuminated, and then to increase slightly again at the offset of the background, finally following the normal course of dark adaptation after the

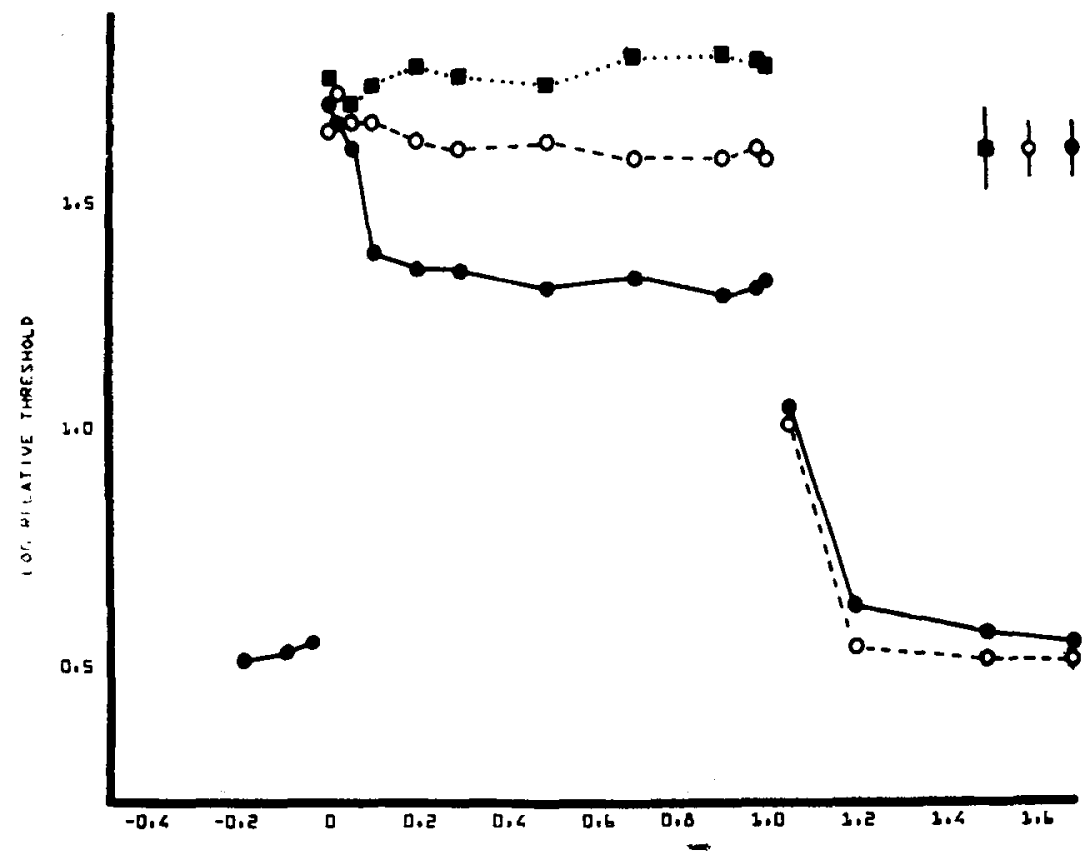

offset of the background. Since this technique described fully the time course of the threshold in relation to the background, it seemed particularly suited to the needs of the present situation. The method will be adapted so that the time course of the probe threshold nay be followed for two types of background condition: (1) where threshold is maximally elevated (as determined from Experiments 1, 2, and 3), and (2) where threshold is relatively stable, that is, for large disk diameters and wide bars. The time course of threshold adjacent to an edge has been described elsewhere by Burkhardt (1966) and Matthews (1968), hence this condition was omitted from the present series of experiments.

\section{EXPERIMENTS 4 AND 5}

In these experiments, the luminance threshold of the $2.5-\mathrm{min}$ test probe is again the dependent variable; there are two independent variables: (1) the type of the background, disk, or bar, and (2) lime between the presentation of the probe and the background. With respect to the latter, the background is presented with a duration of $1 \mathrm{sec}$, as before, but the duration of the test probe is now $10 \mathrm{msec}$. The test probe is presented at varying times before, during, and after the presentation of the background.

The $S$ was first presented with an adapting field of size $9 \times 6 \mathrm{deg}$ and a luminance of $1.9 \mathrm{log} \mathrm{mL}$; the fixation arrangement in this adapting field was the same as in previous experiments, as described in Experiment 1. After an interval of time, the adapting ficld was extinguished and the background was presented to the $S$ for a duration of $1 \mathrm{sec}$. The backgrounds used were: (1) the same size as the adapting field, (2) a disk, 8-min in diam for EL and $11-\mathrm{min}$ in diam for $\mathrm{AH}$. and (3) a bar, of width $8 \mathrm{~min}$ for EL and $11 \mathrm{~min}$ for $\mathrm{AH}$. Different disk and bar widths were used for the two $S$ s because these were the critical widths that gave maximum threshold elevation for each $\mathrm{S}$. Teller (1969) has shown that temporal comparisons are meaningful only when backgrounds are equated for their threshold-raising capacity rather than absolute magnitude. The luminance of all thrce backgrounds was $1.56 \mathrm{log} \mathrm{mL}$. At the end of the I-sec background, the adapting field was again presented, and this remained on until the background was again illuminated on the following trial by the $S$ pressing a button, as before.

A simall 2.5 -min test probe could be presented before, during, or after the presentation of the background. The location of the test probe was at the end of the fixation indicator, as described previously. The background stimuli were carefully centered such that the probe always appeared in the center of the background stimulus. Thus, the probe position was always in the same well-defincd location. The probe was presented for each of the following times: 200,100 , and $50 \mathrm{msec}$ before the onset of the background; $0,20.50,100,200,300$. $500,700,900,980$, and $1,000 \mathrm{msec}$ after the onset of the background; and 50, 200. 500 , and $700 \mathrm{msec}$ after the offset of the background.

The same experimental procedure was used as before, with two modifications. First, on 10 successive trials, the test probe 


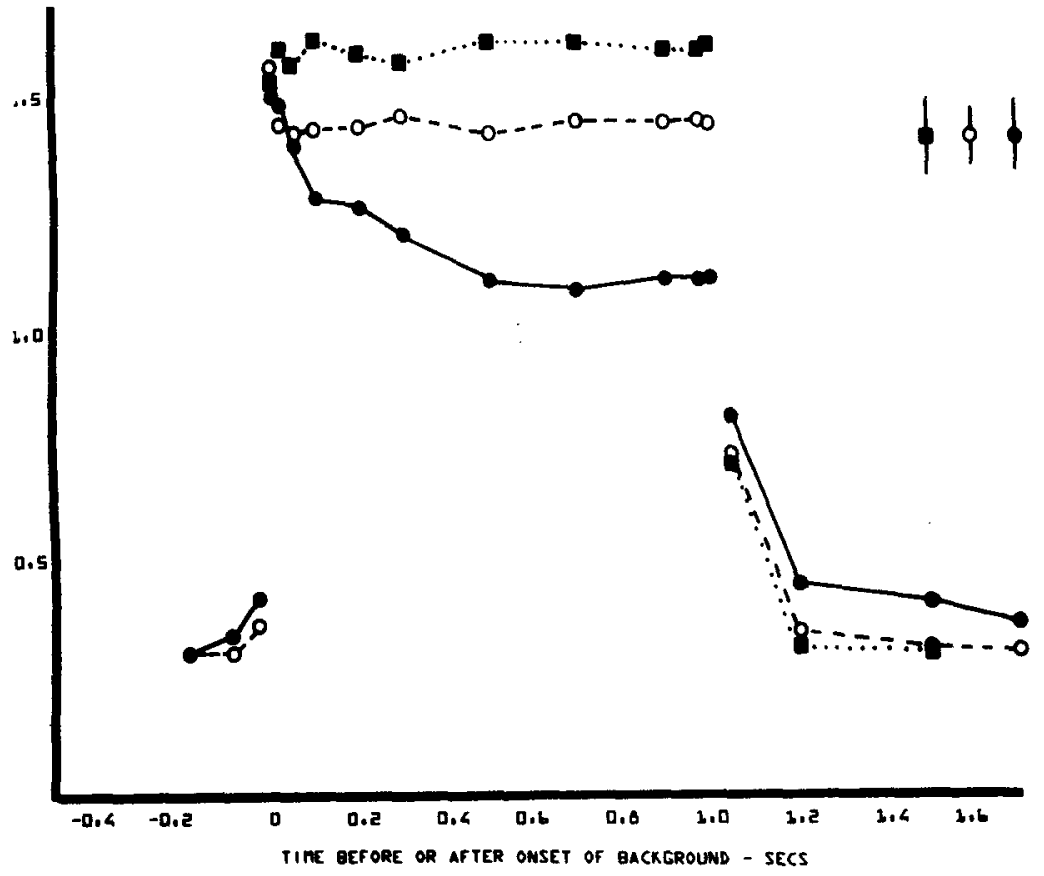

was maintained in the same temporal relationship with the background in order to eliminate effects of temporal uncertainty. Prior to the determination of the threshold on the 10 trials, the test probe was presented in its correct temporal relationship with the background at about $1 \log$ unit above threshold, in order to minimize effects of temporal uncertainty when the testing procedure began. A second modification was that the use of blank trials was eliminated for these experiments. A total of 30 trials was given at each temporal locus of the probe; since only 10 trials were given at a particular time at any one locus, determination of the threshold was accumulated in three sessions for each of the background conditions. These sessions were block randomized between Ss. In any one session, the temporal locus of the test probe was varied randomly between each block of 10 trials. The intertrial interval was at least $30 \mathrm{sec}$ in order to reduce the effects of temporal masking from one background presentation to the next. Viewing conditions were thesame as in the first series of experiments. Threshold was again equated with the luminance that gave $50 \%$ correct detections.

\section{Results}

Figures 4 and 5 indicate results for Ss $\mathrm{EL}$ and $\mathrm{AH}$, respectively. For each $\mathrm{S}$, the increment threshold is plotted as a function of the delay between the onset of the background and the onset of the test probe; negative delays indicate the presentation of the test probe before the background. Background is illuminated at time zero extinguished at $1 \mathrm{sec}$. Increment thresholds are plotted on the same axes for each of the three types of background. Variability is indicated by a plot of one standard deviation for each condition.

The shape of the function obtained with the large uniform background is essentially similar to that obtained by Crawford (1947) and by Matthews (1968). It should be noted that the recovery of sensitivity following the onset of the background follows approximately the same course for the two Ss, although the curve for EL takes a somewhat longer time to flatten out than does that for $\mathrm{AH}$

Again, for the disk and bar backgrounds, the results of the two Ss are essentially similar. It can be seen that, with these stimuli, there is a very rapid recovery of probe threshold to a stable level after the initial peak response at the background onset. This recovery is of the order of $20-50 \mathrm{msec}$ compared with $400-500 \mathrm{msec}$ for the large uniform background.

By comparing the relative time course of threshold in the two situations, it is apparent that the threshold-raising effect of contour adjacency takes time to develop. The effect takes at least $50-100 \mathrm{msec}$ to appear and then a further $300-500 \mathrm{msec}$ to develop fully. These results are essentially similar to those obtained with a single contour background (Matthews, 1968). It should be mentioned here that Burkhardt (1966) has produced somewhat conflicting results using a similar paradigm. In his case, after the background onset, probe threshold adjacent to a step followed a course parallel to the probe threshold located in the center of an
Fig. 5. Description as for Fig. 4. Data for S EL.

extended field. The differences in experimental technique would not appear to be sufficiently great to account for this discrepancy, although the fact that Burkhardt used a vertical line of $1 \times 20 \mathrm{~min}$ subtense as the test probe may be an important factor.

These data give us a more complete understanding of three previously reported spatiotemporal effects in this context, by Westheimer (1967) in the case of disks, Novak and Sperling (1963) in the case of bars, and Matthews (1966) for a single edge. In each of these cases, the threshold-raising effects associated with each of the backgrounds was found to disappear at short background durations below about $50-80 \mathrm{msec}$. The effect was found to gradually appear and increase as the background duration was increased in two of the cases. The present experiments show that it may be possible to equate a background of duration $\mathrm{x}$ msec with the measurement of the probe threshold upon a longer background at $x \mathrm{msec}$ after the background onset. The failure to find the peak at short durations is not due to the fact that the peak takes time to develop, but is a result of the slow recovery of the threshold in locations distant from contours or edges to which the threshold adjacent to the contour is referred.

These data are essentially similar to those presented recently by Teller (1969), using a somewhat analagous paradigm in peripheral vision. In Teller's case, the initial peak threshold was obtained only in situations where the background was extended over somewhat larger areas. There is some indication of this in the present experiments but the data are not clear cnough to adjudicate this point.

With respect to the time course of threshold after the onset of the background, the data suggest that there is a faster recovery of sensitivity in the case of prior stimulation with the disk or bar than with the larger uniform field.

\section{CONCLUSION}

These experiments demonstrate basic similarities in the increment threshold-raising properties of disks, bars. and single edges. The finding that the widths of the disk and of the bar that gave a maximum elevation of probe threshold are almost identical for individual $\mathrm{Ss}$ is strong evidence that the two stimuli are sampling the same type of visual process. The relationship between the double- and single-edge situation is less obvious; however, Experiment 3 gives some 
indication of the manner in which the two may be related. It would appear that the peak threshold obtained with the probe centered on a narrow bar is a direct equivalent of the threshold obtained when the probe is located adjacent to a single edge, at least for the luminances used in these experiments. There has been a recent tradition to treat the threshold-raising capability of a single edge as a separate case or phenomenon; these experiments indicate that such an approach may be prematurely inappropriate.

In terms of the temporal parameters associated with these background stimuli, there is again good evidence to suggest that the similar processes are being sampled in Experiments 4 and 5 . The data are also essentially similar to those obtained with the single edge, using an identical technique (Matthews, 1968). The replication of the temporal course of the increment threshold with the extended backgrounds adds little, of course, to well-established findings and has no bearing on the present arguments. The similarity of the temporal response curve in the situations of the single edge and narrow bar or disk provides the main evidence for the suggestion of similar underlying processing. While all the curves agree that the increment threshold rapidly reaches a stable level after the onset of these backgrounds, there is some disagreement in the nature of the very rapid threshold changes associated with the background onset.

Since Ratliff and Hartline (1959) demonstrated that lateral inhibition in the Limulus eye gave rise to spatial interactions similar to those found in human psychophysics using similar stimulation, perception has become pregnant with lateral inhibitory models for a wide variety of visual phenomena, the range of which has only been matched by the number of untestable assumptions found in some models and an apparent repression of relevant neurophysiological evidence. Together with simple lateral inhibition, the now familiar functional receptive field properties found in a variety of animal visual systems have provided the sensory psychologist with new experimental paradigms with which he hopes he will test basic assumptions of psychophysiological hypotheses. It is the present intention to examine the tenability of simple neurophysiological explanations of the results obtained in these experiments.

Perhaps the simplest line of argument to take would be to assume that each of these phenomena represented some mode of action of lateral inhibition, which by common consensus seems to have its origin in the retina. In the case of the single edge, the similarity between the Limulus work and the increment threshold measures across a luminance step is striking. By assuming that areas subjected to a greater amount of lateral inhibition will have lower thresholds than areas receiving less inhibition, we can satisfy ourselves that this is a parsimonious explanation for the threshold-raising capacity of a single edge. The nature of the mode of action of the lateral inhibition, that is, whether it will serve to reduce the background noise level or change the state of adaptation of the inhibited area, must remain at the moment unanswered. A major objection to the lateral inhibitory explanation for the peak threshold at the single edge has been that the increment threshold fails to take the expected dip in threshold on the darker side of the border that is analogous to the dip in firing rate obtained in Limulus. Fortunately, Wildman (1969) has been able to demonstrate that the increment threshold on the dark side of the border is elevated by scattered light from the brighter side, and hence any threshold depression would be masked. Thus, for the case of the single border, the lateral inhibitory argument has a superficial strength.

The next step will be to attempt to extend this argument to the bar and disk situation. Presumably, the gradual increase in threshold with narrowing bar width and disk diameter is seen as evidence that inhibitory effects in the center of the bar or disk are reduced with a decrease in the area of induction. The failure to find a continued increase in threshold at very small bar widths is considered evidence that there is a small central area over which lateral inhibition does not operate. The reduction in threshold that is then obtained with still smaller backgrounds within these small areas can be accounted for in terms of summation rather than inhibition. This type of argument leads to a view of peripheral processing in terms of a central excitatory area flanked with, or surrounded by, a larger inhibitory area, a notion that, of course, is derived from a wealth of neurophysiological evidence, and gains particular support from electrophysiological data of Baumgartner (1961). The fact that for an equivalent width of background the disk produces a higher peak threshold than the bar fits the model well, since the relative reduction in peax threshold in the bar situation may be attributed to vertical inhibitory influences traveling along the bar; of course, in the disk situation such influences are not possible.

Let us now consider the significance of the findings from Experiments 4 and 5. which investigated the time course of the spatial interactions. The datum of most obvious interest is the recovery of sensitivity of the increment threshold following the onset of the background. Since it is assumed that the low threshold obtained in the center of extended backgrounds in static conditions is due to the maximum lowering effect of the inhibitory interactions, the time course of the increment threshold for these backgrounds should give some indication of the speed with which these interactions develop. The data show that while a small effect develops rapidly, the maximum threshold depression takes from 300 to $500 \mathrm{msec}$ to develop fully after an initial delay of between 50 and $100 \mathrm{msec}$. This may seem to be a rather slow operation to be sccurring at early stages of retinal processing; however, in Limulus it has been shown that the inhibitory influences do not appear until about $100 \mathrm{msec}$ after the impulses that produce them. In addition, Werblin and Dowling (1969) have recently shown that the inhibitory surround found with ganglion-cell receptive fields in the mudpuppy retina takes some time to develop. It would seem, therefore, that the interpretation of the temporal data in terms of the lateral inhibition is not in contradiction with known electrophysiological findings. Some recent suggestions by Smith and Richards (1969) based on psychophysical procedures indicate that lateral inhibition has a propagation velocity of about $0.65 \mathrm{deg} / \mathrm{sec}$; this is certainly faster than a somewhat arbitrary calculation based on the present data, which assume that the crucial area over which lateral inhibition may operate is indicated by the width of bar or disk that produces a leveling of threshold. This would lead to an estimate of a propagation velocity in the region of $0.3 \mathrm{deg} / \mathrm{sec}$; however, this particular calculation should be regarded as no more than an academic exercise, since the ground rules necessary for the assumptions involved in the calculation have no precedent.

Thus, the relatively simple model of lateral inhibition/receptive field organization appears to account well for these findings and involves a minimum number of discrepancies between electrophysiological and psychophysical evidence. However, there are at least two serious criticisms of the model that would seem to reduce its general applicability to these situations. First, to what extent do eye movements that would seem to be present in all these experiments confound the model? The lateral inhibitory model is based upon a fixed eye, whereas in the experimental situation transient effects are produced when the small eye movements that generally accompany fixation permit 
the eye to cross areas of differing luminance. The traditional method of testing the role of eye movements has been either to reduce exposure time to perhaps less than $10 \mathrm{msec}$ or to use a stabilized-image technique. In the case of the threshold-elevation effects associated with each of the backgrounds used in this study, stabilizing the retinal image is found to make no difference to the results in the case of disks (Teller, Andrews, \& Barlow, 1966). On the other hand, using the bar background, Teller (1968) failed to find threshold elevation with small bar widths when the image was stabilized. For the single edge the relevant experiment has yet to be performed. Teller's (1968) result would seem to cast some doubt both on my argument that similar processing is involved in each of these situations and to throw further doubt on a simple argument for lateral inhibition. It should be noted, however, that in Teller's experiment black bars were used on a background of higher luminance, compared with bright bars on a darker background in the present experimental design. Experiments that eliminate eye movements with brief exposures can have no adjudicating role in this argument, since the data from Experiments 4 and 5 show that the threshold-raising effect should not occur at short exposure durations as Novak and Sperling (1963), Matthews (1966), and Westheimer (1967) have shown. Whether the failure to obtain the effect is due to the elimination of eye movements or due to the latency and slow development of the inhibitory process must remain an open and crucial issue at the present time, although I feel the available evidence favors the latter explanation.

The second argument against a lateral inhibitory interpretation stems from the finding that there is no continual decrease in threshold as the width of the bar or disk is made progressively larger. Since the amount of inhibition generated is assumed to be proportional to the spatial extent of the inducing area, one would expect a continued reduction in threshold. However, for the $S$ s in this experiment, the threshold leveled out with background widths beyond $15 \mathrm{~min}$ of arc. A counter to this argument would be to invoke the concept of disinhibition such that an increase of inducing area beyond a certain critical value leads to inhibition of inhibition, which might produce a stabilizing effect on threshold. Such effects are known to exist in the Limulus eye, but would require a demonstration of spatial disinhibition in the human visual system to validate this counter argument adequately.

A third possible argument against the lateral inhibition explanation comes from experiments with dichoptic presentation of background and probe. For the case of bars, Novak (1967) has shown that the threshold-elevating effects associated with the edges of dark bars cannot be found when the probe and bar are presented to different eyes. However, Markoff and Sturr (1969) have demonstrated that the threshold-elevating capacity of small disks may be found with dichoptic presentation. The argument for the dichoptic experiment as an adjudicator between central and peripheral processing has never been convincing, in that it has often been used to assume that when similar results are obtained with dichoptic and monoptic stimulation then there is some indication of central processing. This is to ignore the possibility that either particular patterns of information processing may occur at different levels in the visual system or that a particular processing pattern is "held" in a particular form such that it may be operated upon at other levels in the system. Thus, the apparent discrepancy between the results of Novak and Markoff and Sturr are not necessarily damaging for the arguments presented above. However, these discrepancies do require modifications to the development of such arguments, but at the present time would require the introduction of untried and at present untestable concepts.

In general, the evidence from the five experiments leads to a compelling argument that similar processes are involved when the increment threshold of a small probe is elevated by a small disk, narrow bar, or the proximity of a singie luminance gradient. A requirement of this assumption is that a manipulation of a particular variable will have analogous effects for the three background conditions. However, both the stabilized image technique and dichoptic presentation do not produce similar effects. These results do not detract from the argument at the present time, since experimental conditions between laboratories are not identical. The use of neurophysiological concepts such as lateral inhibition and functional receptive field organization produces a simple model that is able to account for the majority of the data. In the general case, lateral inhibition serves to account for these phenomena in the simplest manner, and it is to be hoped that outstanding discrepancies can be resolved by future experimentation. However, the very simplicity of such concepts should not lead us to use them in unwarranted situations where their value will be quickly lost if called upon 10 account for every masking phenomenon that the experimental psychologist discovers.

\section{REFERENCES}

BAUMGARTNER, G. Kontrastlichteffekte an retinalen Ganglicnzellen: Ableitung vom Tractus opticus der Katze. In R. Jung and $\mathrm{H}$. Kornhuber (Fids.), Ncurophysiologie und Psychophysik des visuellen Systems Burlin: Springer-Verlag, 1961. Pp. 45.53.

BURKHARIT, D. A. Brightness and the increment threshold. Journal of the Optical Society of America, 1966. 56, 979-981.

( $R A$ WF ORD, B. H. Visual adaptation in relation to brief conditioning stimuli. Proceedings of the Royal Society (London), 1947. 134B. 283-300.

CUTRONA, L. J.. JR., \& RICHARDS, W. I ovcal and parafoveal anisotropics in human photopic receptive field structure. Paper Thl:20 presented to Optical Society of America, San Diego, March 1969.

I. IORENTINI, A., JYANNE, M., \& TORALDO DI FRANCIA, G. Measurements of differential threshold in the presence of a spatial illumination gradient. Atti della Fondazioni Giorgio Ronchi. 1955. 10, 371-379.

IORENTINI, A., \& ZOLI, M. T. Detection of a target superimposed to a step pattern of jllumination. Atti della londazioni Giorgio Ronchi, 1966, 21, 338-356.

MARKOFF, J. I. \& STURR, J. F. Monoptic and dichoptic measures of the increment threshold. Paper l:D15 presented to the Optical Society of America, Chicago, October 1969.

MATTHEWS. M. L. Appearance of Mach bands for short durations and at sharply focused contours. Journal of the Optical Society of America. 1966. 56. 1401-1402.

MATTHEWS, M. L. Mach bands: A psychophysical investigation. Unpublished doctoral thesis, University of Nottingham. 1967.

MATTHEWS, M. L. Some temporal aspects of visual spatial interactions associated with illumination gradients. Nature. 1968. 218, 1061.1063.

NOVAK, S. Invariance of detection thresholds across a light-dark boundary using dichoptic presentation. Journal of the Optical Society of Ainerica, 1967.57, 1059-1060.

NOVAK. S., \& SPERLING, G. Visual thresholds near a continuously visible or briefly presented light-dark boundary. Optica Acta, 1963, 10 187-191.

RATLIFF, I:. \& HARTLINE, H, K. The response of limulus optic nerve fibers to patterns of illumination on the receptor mosaic. Journal of Cieneral Physiology. 1959. $42,1241 \cdot 1255$.

RATOOSH. P.. \& GRAHAM, C. H. Areal effects in foveal brightness discrimination. Journal of lixperimental Psychology. 1951, 42, 369-375.

SII:CI:L, M. H. A note on luminance control in tachistoscopes. Behavior Research Mcthods \& Instrumentation, 1968, 1, 33.

SMITH. R. A.. \& RICHARDS. W. Propagation velocity of lateral interaction in the human visual system. Journal of the Optical Society of America. 1969, 59, 1469-1472.

TILLLER. D. Y. Increment thresholds on black bars. Vision Research. 1968, 8, 713-718.

TIILI.ER, D. Y. Visual sensitization by annular surrounds. Paper ThH14 presented to Optical Socjety of America. San Diego. March 1969. TLLI.I.R. D. Y., ANDREWS. D. P.. \& BARLOW H. B. Local adaptation in stabilized vision. Vision Recearch, 1966. 6. 701.705.

WI.RBIIN. 1. S., \& DOWLING, J. E: Organization of retina of the mudpuppy, Nectumes maculosus. II. Intracellular recording. Journal of Neurophysiology. 1969. 32 $3.39-355$ 
WISSTHEIMER, G. Spatjal interaction in the human retina during scotopic vision. Jouma of Physiology, 1965, 181, 881-894.

WESTHEIMER, G. Spatial interaction in human cone vision. Journal of Physiology, 1967, 190. 139-154.

WILDMAN, K. N, Visual sensitivity at an edge.

Paper presented to Psychonomic Society,
St. Louis. November 1969.

(Accepted for publication April 23. 1970.) 